\title{
Correlations between microbiota and metabolites after faecal microbiota transfer in irritable bowel syndrome
}

\author{
S. Holster ${ }^{1 *}$, D. Repsilber ${ }^{1}$, D. Geng ${ }^{2}$, T. Hyötyläinen ${ }^{2}$, A. Salonen ${ }^{3}$, C.M. Lindqvist ${ }^{1}$, S.K. Rajan ${ }^{1}$, W.M. de Vos ${ }^{3,4}$, \\ R.J. Brummer ${ }^{1}$ and J. König ${ }^{1^{*}}$ \\ ${ }^{1}$ Nutrition-Gut-Brain Interactions Research Centre, Faculty of Medicine and Health, School of Medical Sciences, Örebro \\ University, Örebro, Sweden; ${ }^{2}$ Man-Technology-Environmental Research Centre, Faculty of Business, Science and Engineering, \\ School of Science and Technology, Örebro University, Örebro, Sweden; ${ }^{3}$ Human Microbiome Research Program, Faculty \\ of Medicine, University of Helsinki, Helsinki, Finland; ${ }^{4}$ Laboratory of Microbiology, Wageningen University and Research \\ Centre, Wageningen, the Netherlands; savanneholster@live.nl; julia.konig@oru.se
}

Received: 16 January 2020 / Accepted: 3 August 2020

(c) 2020 Wageningen Academic Publishers

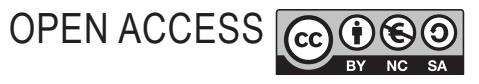

RESEARCH ARTICLE

\begin{abstract}
Faecal microbiota transfer (FMT) consists of the infusion of donor faecal material into the intestine of a patient with the aim to restore a disturbed gut microbiota. In this study, it was investigated whether FMT has an effect on faecal microbial composition, its functional capacity, faecal metabolite profiles and their interactions in 16 irritable bowel syndrome (IBS) patients. Faecal samples from eight different time points before and until six months after allogenic FMT (faecal material from a healthy donor) as well as autologous FMT (own faecal material) were analysed by $16 \mathrm{~S}$ RNA gene amplicon sequencing and gas chromatography coupled to mass spectrometry (GS-MS). The results showed that the allogenic FMT resulted in alterations in the microbial composition that were detectable up to six months, whereas after autologous FMT this was not the case. Similar results were found for the functional profiles, which were predicted from the phylogenetic sequencing data. While both allogenic FMT as well as autologous FMT did not have an effect on the faecal metabolites measured in this study, correlations between the microbial composition and the metabolites showed that the microbe-metabolite interactions seemed to be disrupted after allogenic FMT compared to autologous FMT. This shows that FMT can lead to altered interactions between the gut microbiota and its metabolites in IBS patients. Further research should investigate if and how this affects efficacy of FMT treatments.
\end{abstract}

Keywords: microbe-metabolite interactions, colonic microbiota, metabolite profiles, faecal microbiota transplantation

\section{Introduction}

Faecal microbiota transfer (FMT) consists of the infusion of faecal material from a healthy donor into the intestine of a patient with the aim to restore the disturbed faecal microbiota of the recipient. FMT is found to be a highly effective treatment in patients with recurrent Clostridioides difficile infection (CDI) (Quraishi et al., 2017). Additionally, it has been studied as a potential treatment for ulcerative colitis (UC) (Costello et al., 2019; Moayyedi et al., 2015; Paramsothy et al., 2017; Rossen et al., 2015), metabolomic syndrome (Kootte et al., 2017; Vrieze et al., 2012) and irritable bowel syndrome (IBS) (Halkjaer et al., 2018; Holster et al., 2019b; Johnsen et al., 2018).
Microbes are the major component transferred in FMT (De Vos, 2013) and it has been shown that FMT results in changes of the microbial composition in the intestinal tract that can persist for months (Li et al., 2016). While these new microbes may interact directly with the host, it is likely that it is their metabolic activity that has an impact on the gastrointestinal ecosystem. Hence, the produced metabolites are an important link between the gut microbiota, the colonic mucosal response and, potentially, the immune homoeostasis and health of the host (Levy et al., 2016). Nevertheless, only few studies have investigated the impact of FMT on gut microbiota metabolites. The metabolites found in the highest amounts are short chain fatty acids (SCFAs) and branched chain fatty acids (BCFAs), 
mainly derived from undigested dietary carbohydrates and proteins, respectively (Scott et al., 2013). SCFAs (acetate, propionate and butyrate) are essential for the homeostasis of the host (Van der Beek et al., 2017). For instance, butyrate is the main energy source for colonocytes and has shown to have anti-inflammatory (Correa-Oliveira et al., 2016) and anti-carcinogenic effects (Wu et al., 2018), as well as having the ability to decrease visceral sensitivity in healthy subjects (Vanhoutvin et al., 2009). In addition, the gut microbiota can affect the metabolism of dietary amino acids and is able to synthesise amino acids de novo (Lin et al., 2017). Faecal amino acids have been shown to be more abundant in colorectal cancer (Yang et al., 2019), and amino acid profiles in serum and plasma could be used to distinguish between IBD and healthy subjects (Schicho et al., 2012). The most abundant amino acids within the gut microbiota are branched chained amino acids (BCAA) (Dai et al., 2011), and these have shown to be harmful when present in high amounts in the systemic circulation (Neis et al., 2015). Serum levels of these amino acids were found to be associated with the aberrant microbiota of subjects with insulin resistance, possibly providing a link between the gut microbiota and insulin sensitivity (Pedersen et al., 2016). Hence, profiling these gut microbiota-derived metabolites might give us an important insight into the function of the gut microbiota and their integration with the host environment.

We have previously reported the effects of allogenic FMT (faecal material from a healthy donor) as well as autologous FMT (own faecal material) on the symptoms and faecal microbiota of IBS patients based on phylogenetic microarray analysis two and eight weeks after FMT compared to baseline (- two weeks sample) (Holster et al., 2019b). Here, we extend the faecal microbial composition data with samples from one day, three days, seven days, four weeks, eight weeks and six months using $16 \mathrm{~S}$ rRNA gene amplicon sequencing. In parallel, faecal metabolites were assessed with gas chromatography coupled to mass spectrometry (GS-MS) in samples from the same time points. The aim of this study was to investigate the effect of FMT on both faecal microbiota composition and functional profiles as well as the associated microbiota-produced metabolites (e.g. SCFAs, BCFA and amino acids), and patterns of their interactions.

\section{Methods}

\section{Study design}

As described in our recent publication, we treated 17 IBS patients with either FMT from a healthy donor (allogenic FMT, $\mathrm{n}=8$ ) or FMT with their own stool (autologous FMT, $\mathrm{n}=9$ ) (Holster et al., 2019a). The faecal transplant (30 g of stool in $150 \mathrm{ml}$ sterile saline and $10 \%$ glycerol) was administered into the caecum by whole colonoscopy after bowel cleansing. One of the patients in the autologous FMT group decided not to continue with the study after receiving the FMT. Hence, faecal samples from 16 patients were collected two weeks and one day before FMT (baseline) and three days, seven days, two weeks, four weeks, eight weeks and six months after FMT. The study was conducted according to the principles of the Declaration of Helsinki and its revisions, and ethical approval was obtained from the Central Ethical Review Board of Uppsala, Sweden (registration number 2013/180). The trial has been registered at ClinicalTrials.gov (NCT02092402) on March 20, 2014.

\section{Faecal microbial DNA extraction and microbiota analysis}

Microbial DNA from faecal samples was isolated using repeated bead beating (Salonen et al., 2010) and the QIAamp DNA stool extraction kit (Qiagen, Hilden, Germany) according to the manufacturer's instructions. DNA concentration was assessed using a Qubit spectrometer (Thermo Fisher Scientific, Boston, MA). The samples were subjected to $16 \mathrm{~S}$ rRNA gene V3-V4 PCR amplification and the amplicons were indexed and characterised by next generation sequencing (NGS) using an Illumina HiSeq platform (Illumina, San Diego, CA, USA) as previously described (Virtanen et al., 2019) in order to assess changes in faecal microbiota composition. To assess the microbiotic profiles sequencing reads were processed with Mothur v. 1.35.1 using Greengenes version 13.5, containing OTUs clustered at $99 \%$ sequence identity, as the reference database (Schloss et al., 2009). Taxonomic classification on species level were used in further analyses.

\section{Faecal metabolite extraction and quantification}

One aliquot of $100 \mathrm{mg}$ faecal material was used for dry weight determination and one for SCFAs and amino acid extraction. $1 \mathrm{ml}$ of $5 \mathrm{mM}$ aqueous $\mathrm{NaOH}$ containing internal standard $(5 \mu \mathrm{g} / \mathrm{ml}$ caproic acid-d $3+5 \mu \mathrm{g} / \mathrm{ml}$ valine-d) was added to the $100 \mathrm{mg}$ aliquot for SCFAs and amino acid extraction. The sample was homogenised with a micropestle and mixed for $10 \mathrm{~min}$ on a shaker (300 rpm). After shaking, the sample was centrifuged for $20 \mathrm{~min}$ at $13,200 \times \mathrm{g}$ at $4{ }^{\circ} \mathrm{C}$. $300 \mu \mathrm{l}$ of MQ water, $500 \mu \mathrm{l}$ propanol/pyridine mixture solvent ( $\mathrm{v} / \mathrm{v}=3: 2), 100 \mu \mathrm{l}$ of propyl-chloroformate were added to $500 \mu \mathrm{l}$ of faecal water obtained after centrifugation. The sample was vortexed and ultrasonicated for $1 \mathrm{~min}$. After adding $300 \mu \mathrm{l}$ of hexane, the sample was vortexed and centrifuged for $5 \mathrm{~min}$ at 2,000 $\times \mathrm{g}$. $300 \mu \mathrm{l}$ from the hexane layer was collected in a GC vial and the sample was reextracted with $200 \mu \mathrm{l}$ hexane, and retention index standards were added before analysis. The chemicals used for metabolites analysis were purchased from Sigma-Aldrich (St. Louis, MO, USA). The following metabolites in the faecal samples were measured by GC: acetic acid, propionic acid, isobutyric acid, butyric acid, 
2-methylbutyric acid (2-MBA), isovaleric acid, valeric acid, methione, tyrosine, valine, leucine and isoleucine. Internal standards were used for quantification: valine $\mathrm{D}_{8}$ and hexanoic acid $\mathrm{D}_{4}$. Undecane and tridecane were used as retention time index standards (RI). The aliquots with $100 \mathrm{mg}$ faecal sample for dry weight determination were freeze-dried overnight at $-50{ }^{\circ} \mathrm{C}$.

The measurements of metabolites were performed on a HP $6890 \mathrm{~N}$ gas chromatography coupled to HP 5973 single quadrupole mass spectrometry (GC-MS) (Agilent Technologies, Santa Clara, CA, USA). The GC oven temperature was as follows: $45{ }^{\circ} \mathrm{C}(4 \mathrm{~min}) ; 10{ }^{\circ} \mathrm{C} / \mathrm{min}$ to $70{ }^{\circ} \mathrm{C} ; 3{ }^{\circ} \mathrm{C} / \mathrm{min}$ to $85{ }^{\circ} \mathrm{C} ; 5{ }^{\circ} \mathrm{C} / \mathrm{min}$ to $110{ }^{\circ} \mathrm{C}$; $30{ }^{\circ} \mathrm{C} / \mathrm{min}$ to $290{ }^{\circ} \mathrm{C}$ (8 min). Splitless injections of $1 \mu$ of the final extract and using a single taper deactivated liner (Agilent Technologies), were carried out at $260^{\circ} \mathrm{C}$ onto a DB-5MS (5\% diphenyl-95\% dimethylpolysiloxane), $30 \mathrm{~m} \times 0.25 \mathrm{~mm} \times$ $0.25 \mu \mathrm{m}$ (Agilent Technologies). Helium was used as carrier gas at a constant flow rate of $1.0 \mathrm{ml} / \mathrm{min}$. MS measurements were performed in the selective ion monitoring (SIM) mode. The ions monitored for the analytes are shown in Supplementary Table S1.

\section{Gene expression colonic transporters}

Expression of the colonic butyrate transporters (MCT1 and SMCT1), subunits of the large neutral amino acid transporter (LAT1), the serotonin transporter (SERT) and the folate carrier (RFC1) was measured with qPCR. Colonic biopsies were collected from the uncleansed sigmoid (20$25 \mathrm{~cm}$ from the anal verge, at the crossing with the arteria iliaca communis) at baseline, two weeks and eight weeks after FMT. RNA was isolated as previously described (Holster et al., 2019a). cDNA was synthesised according to the protocol of the Superscript ${ }^{\mathrm{tm}}$ VILO ${ }^{\mathrm{mw}} \mathrm{cDNA}$ synthesis kit (ThermoFisher Scientific, Waltham, MA, USA). The following Taqman primer-probe sets (Life Technologies, Carlsbad, CA, USA) were used for the amplification of each colonic transporter by RT PCR: SLC5A8 (SMCT1, Hs00377618_m1), SLC16A1 (MCT1, Hs01560299_m1), SLC3A2 (LAT1 heavy chain, Hs00374243_m1), SLC7A5 (LAT1 light chain, Hs01001189_m1), SLC3A2 (LAT1 heavy chain, Hs00374243_m1), SLC6A4 (SERT, Hs00984349_ m1), SLC19A1 (RFC1, Hs00953344_m1) and GAPDH (Hs03929097_g1). The thermal cycling conditions of the Applied Biosystems 7900HT Fast Real-Time PCR system (Life Technologies) were set to $95^{\circ} \mathrm{C}$ for $20 \mathrm{~s}$, followed by 40 cycles at $95^{\circ} \mathrm{C}$ for $2 \mathrm{~s}$, ended by one cycle of $60^{\circ} \mathrm{C}$ for $20 \mathrm{~s}$ and then hold at $4{ }^{\circ} \mathrm{C}$. GAPDH was used as reference gene for each sample. The relative gene expression of the colonic transporters was calculated using the comparative 2- $\Delta \Delta \mathrm{Ct}$ method (Livak and Schmittgen, 2001), and was normalised to GAPDH expression.

\section{Data analysis}

\section{Mixed-effect analysis of the faecal metabolites}

The faecal metabolites were analysed using a mixedeffects linear model analysis as implemented in Graphpad Prism 8.0. This mixed model uses a compound symmetry covariance matrix and Geisser-Greenhouse correction and is fitted using Restricted Maximum Likelihood (REML). The Sidak's multiple comparisons test was used to correct for multiple comparisons.

\section{Principle Coordinate Analysis}

Principle Coordinate Analysis ( $\mathrm{PCoA}$ ) has proven a flexible tool for coordination analysis of microbiota composition data (Hout et al., 2013; Kenkel and Orloci, 1986; NavasMolina et al., 2013) to detect overall effects of treatments on composition of microbiota. We implemented it by using function 'cmdscale' as part of the R-language. For analysing composition data, as in the microbiota compositional profiles, a specific distance measure, Aitchison distance, has been shown to be especially appropriate (Aitchison and Greenacre, 2002). In our analysis, we implemented R-package 'coda.base' for using it (Comas-Cufi, 2019).

\section{Microbial functional prediction}

The NGS data was used to predict the functional capacity of the microbiota found at the time points before and after FMT. Functions were predicted using PICRUSt version 1.1.1 using Greengenes database version 13.5 and default settings (Langille et al., 2013). The predicted functions are given as profiles of KEGG pathways (Kanehisa and Goto, 2000).

\section{Trajectory analysis of microbiota composition, metabolites and functional prediction profiles}

Sparse Partial Least Squares (sPLS) regression was employed for visualising the changes over time of treated individuals' microbiota composition, metabolites and functional prediction profiles across the two baseline and six follow-up time points. The sPLS model was used to describe how bacterial composition, metabolites and bacterial predicted functions varied over time, separately for subjects treated with allogenic and autologous FMT. The sPLS latent variables, hence, represent those parts of varying bacterial composition, metabolites or predicted functions which vary with time in a similar matter for all subjects in the respective group. Function 'spls' from R-package 'mixOmics' (Cao et al., 2017; Rohart et al., 2017) was used for this analysis, choosing ncomp $=2$ components and keepX $=10$ allowed variables in each component. For microbiota composition, metabolites and predicted functions, respective parts of the data (e.g. baseline versus later timepoints) were characterised by their mean values 
(centre of ellipses) and variance-covariance structure (axes orientation and dimensions of the displayed ellipses). This allows comparing location and variance without claiming to give $95 \%$ confidence intervals of the means, as these are not as easily computed and interpreted for data with timeseries and repeated values structure.

\section{Partial correlation analyses of interactions between microbiota and metabolite time courses}

Pairwise shrunk partial correlations were computed for all pairs of microbiota compositional features, i.e. relative abundance of genera and species, and the faecal metabolite profiles (function 'cor2pcor' from R-package 'corpcor' (Schafer and Strimmer, 2005; Schafer et al., 2017)). Using a cut-off of $\rho>0.4$, partial correlation network graphs for the interactions between microbiota species (genus) and metabolites were produced.

\section{Multivariate correlation structures between microbiota and metabolites}

Sparse canonical correlation analysis (function 'CCA' from R-package 'PMA' (Witten et al., 2009)) was employed to characterise the crosstalk of microbiota species and faecal metabolites across the time series, for each of the individuals in our study. In order to maintain the impact of the large proportional differences in microbiota composition between the different microbiota species (genus) as well as metabolite levels, the analyses were run without prior standardisation of either microbiota or metabolite time series profiles. Multivariate correlations of the first components from the canonical correlation analysis are reported as single values together with boxplots. These analyses were performed for correlating all measured metabolites to either all microbiota composition (genus) profiles or to the predicted functional profiles (KEGG pathways). In addition, we focused on specific pathways which represent either mainly saccharolytic or proteolytic metabolic processes, and investigated their correlation pattern with those measured faecal metabolites in our study which can be grouped as to belong to these processes.

\section{Results}

\section{Next generation sequencing microbiota composition data}

Supplementary Figure S1 shows the top 10 most abundant genera in the faecal samples of patients before and after allogenic or autologous FMT. Microbiota compositional profiles obtained by NGS were analysed separately in the allogenic and in the autologous group using both unsupervised (PCoA, Figure 1) and supervised (sPLS, Figure 2 ) ordination methods. The PCoA analysis shows that the microbiota of each individual clusters together, even after receiving allogenic FMT. However, the samples after allogenic FMT are separated from the baseline samples in most of the individuals (Figure 1A), which is not the case after autologous FMT (Figure 1B). This indicates, as to be expected, that the gut microbiota in the IBS patients after FMT from a healthy donor changes more than after receiving their own stool back. When focusing on the differences between the time points (see the sPLS plots in Figure 2) it appears that the faecal microbiota of the subjects that received allogenic FMT (Figure 2A) separates more clearly from their baseline samples than is the case for the autologous FMT group (Figure 2B). This confirms further that the allogenic FMT causes a long-term colonisation of newly introduced microbes in the IBS subjects that lasts at least six months.

Some of the six-month samples in the autologous group show a very distinct separation from the other samples (Figure 2B). The autologous FMT did not result in improved symptoms (Holster et al., 2019b), hence, subjects in this group were more likely to change their diet and/ or medication, even if asked not to during the course of the study. As these changes could have had large effects on the microbiota composition, they were excluded in a separate analysis, however, similar results were obtained (see Supplementary Figure S2).

In addition, Figure 2 shows that there are differences in microbiota composition between subjects. Apart from known differences in microbiota composition in IBS patients (Pittayanon et al., 2019), this could be due to the temporal instability of the microbiota in IBS patients (Durban et al., 2013; Matto et al., 2005) or a change in symptoms over time. However, in general, there does not seem to be a clear relationship between improvement of symptoms and change in microbiota after FMT (Halkjaer et al., 2018; Lahtinen et al., 2020).

\section{Faecal metabolites}

Figure 3 shows the concentrations of the metabolites measured in faecal samples before and after FMT in the allogenic and autologous FMT group, corrected for baseline values. No large differences between the allogenic and autologous FMT group were observed. Butyric acid was significantly increased three days after FMT in the autologous group (mixed-effect analysis with Sidak multiple comparisons test, $P<0.01$ ), which is probably due to the differences in the absolute values at baseline (see Supplementary Figure S3). Similar results were obtained when the metabolite concentrations were normalised to faecal dry weight (Supplementary Figure S4 and S5). Sparse PLS analyses were not able to separate the different time points from each other (Figure 4), contrary to what was shown for the microbiota composition data. These results show that although the microbiota changes after allogenic FMT, the metabolites measured in this study did not. 
A

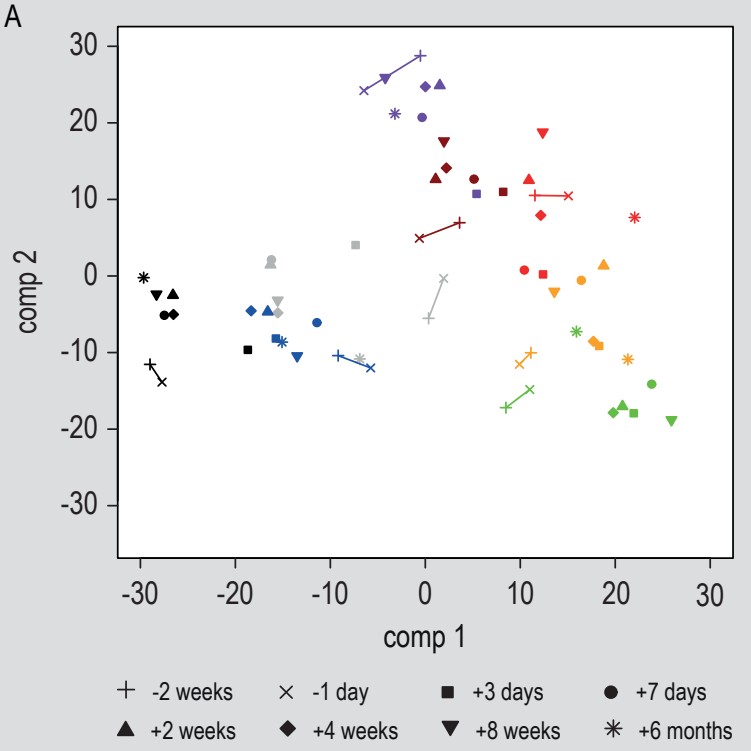

B

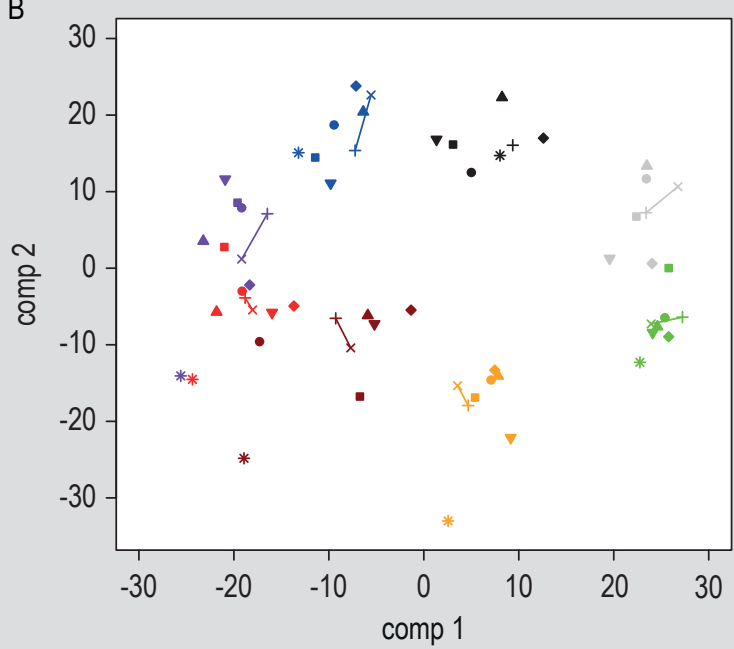

Figure 1. Principal component analysis score plots of faecal microbial composition within the allogenic (A) and autologous group (B) at different time points before and after faecal microbiota transfer (FMT). The first and second component are shown on the $\mathrm{x}$ - and $\mathrm{y}$-axis. Colours indicate the different subjects and symbols represent time points. The time points before FMT (-2 weeks and -1 day) are connected with a line in order to visualise that the samples after allogenic FMT were separated from the baseline samples in most participants. In the autologous FMT group the samples after FMT were less separated from the corresponding baseline samples.

A

莡

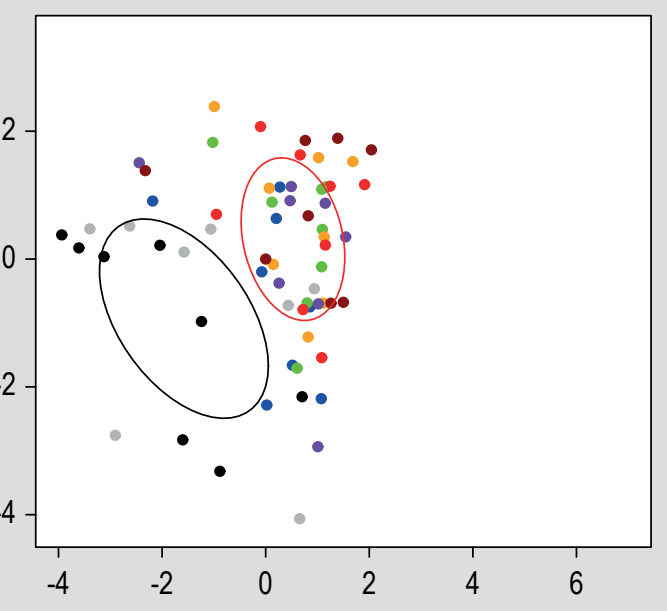

latent var 1
B

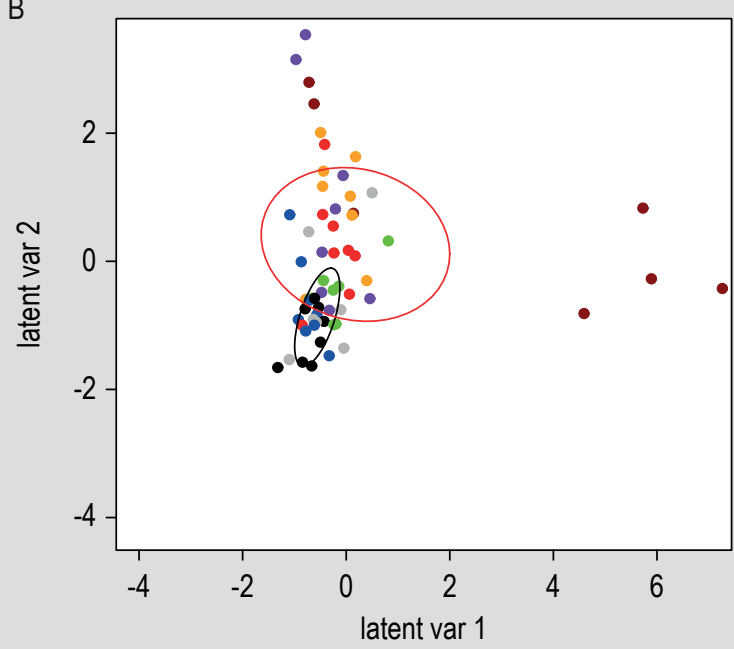

Figure 2. Sparse partial least squares score plots of the microbiota composition of faecal microbial composition within the allogenic (A) and autologous group (B) at different time points before and after faecal microbiota transfer (FMT). The first and second latent variable are shown on the $\mathrm{x}$ - and $\mathrm{y}$-axis. Different colours indicate different time points. Ellipses represent location and dimensions of mean values and covariance structure of the respective part of the data. The black ellipse represents the samples before FMT ( -2 weeks and -1 day samples) and the red ellipse represents the samples after FMT ( 3 days, 7 days, 2 weeks, 4 weeks, 8 weeks and 6 months). The ellipses show that the microbiota samples were clearly separated from the baseline samples after allogenic FMT, which was not the case after autologous FMT. 


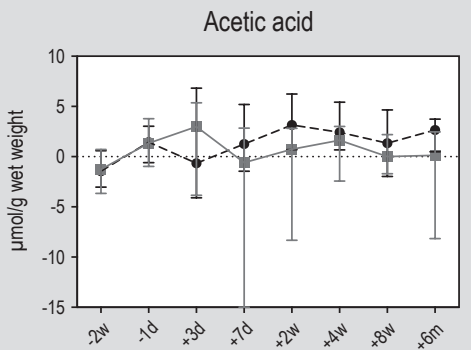

$$
\text { - Allogenic } \quad-\text { Autologous }
$$
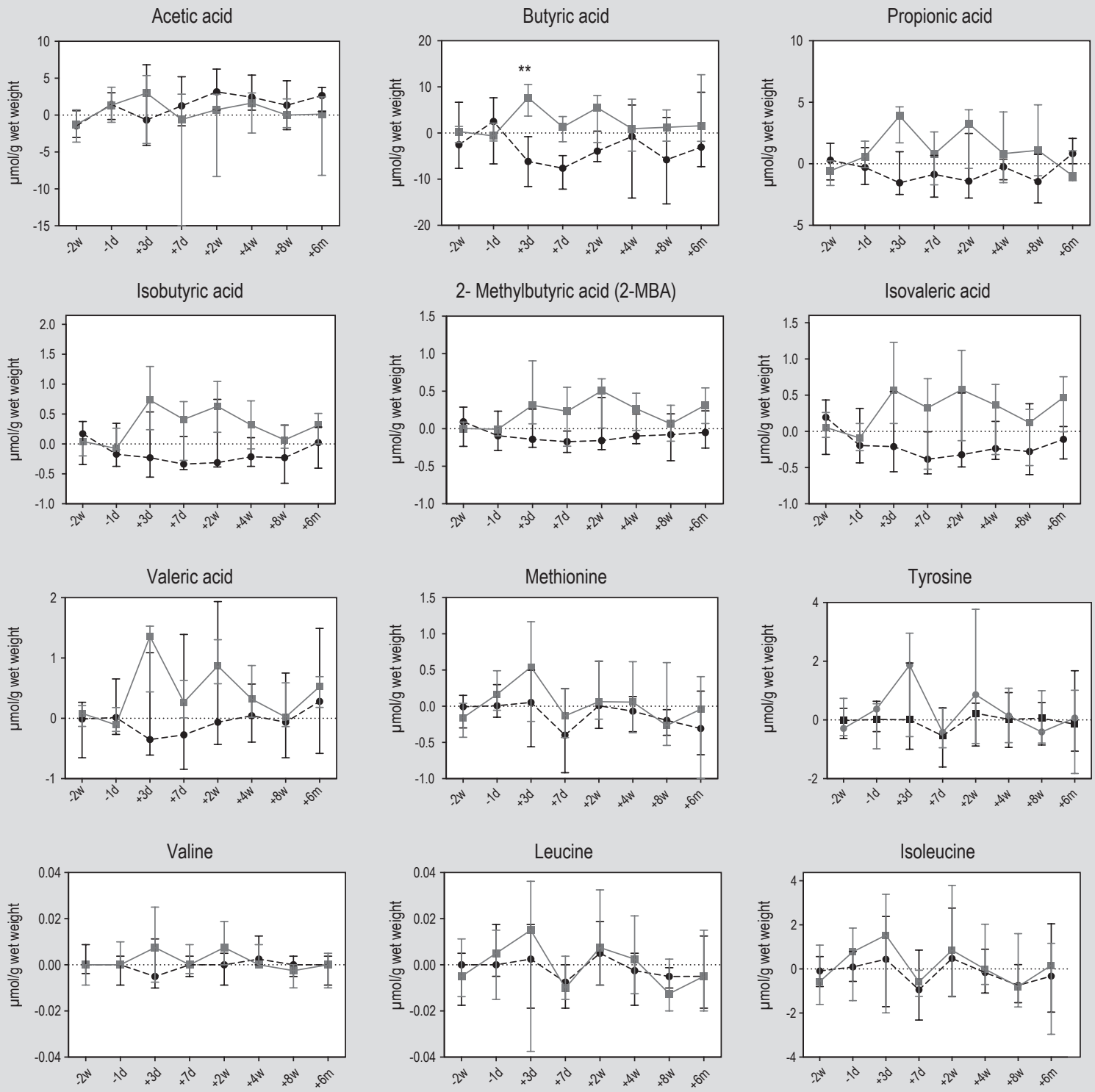

Figure 3. Baseline-corrected concentrations of different metabolites in faecal samples before and after allogenic or autologous faecal microbiota transfer in irritable bowel syndrome patients. The average of the concentrations from two weeks and one day before treatment were subtracted from the concentrations at all time points. ${ }^{* *}$ indicates $P<0.01$. Median values with interquartile range are shown. The absolute data can be found in Supplementary Table S2. D=days, w=weeks, $m=$ months.

\section{Correlations between microbiota and metabolites in faecal samples}

\section{Shrunk partial correlations}

In order to investigate the interactions between the microbiota composition and the metabolites measured in the faecal samples, correlation analyses were performed. Pairwise correlations between single faecal microbiota taxa profiles and specific metabolite profiles were assessed as shrunk partial correlations in order to overcome the imbalance of a large number of variables compared to a small number of samples, which does not allow for the classical approach of computing partial correlations. Analysis of shrunk partial correlations between individual faecal microbiota compositions and the measured faecal metabolites resulted in a smaller number of relevant correlations (a cut-off of $\rho>0.4$ ) in the allogenic FMT group compared to the autologous FMT group (Figure $5 \mathrm{~A}$, Supplementary Table S2). These results indicate that the correlations present after autologous FMT, which is considered the control group, are disrupted after 
A

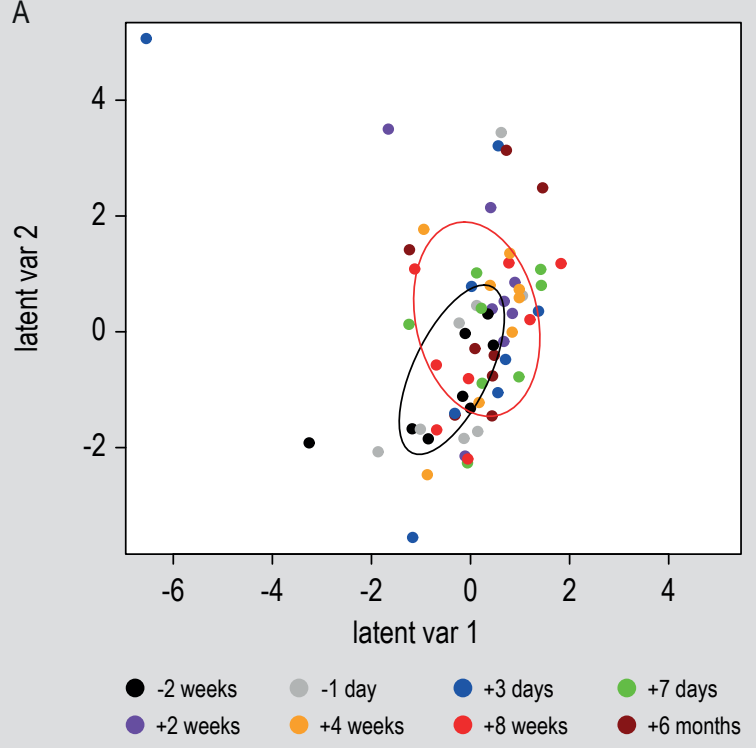

B

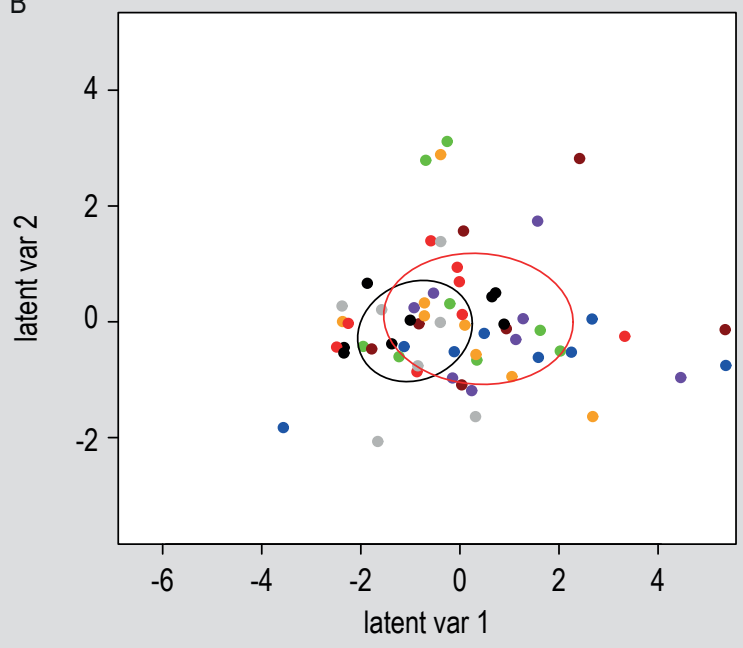

Figure 4. Sparse partial least squares score plots of measured metabolites within the allogenic group (A) and autologous group (B) at different time points before and after faecal microbiota transfer (FMT). The first and second latent variable are shown on the $\mathrm{x}$ - and $\mathrm{y}$-axis. Different colours indicate different time points. Ellipses represent location and dimensions of mean values and covariance structure of the respective part of the data. The black ellipse represents the samples before FMT (-2 weeks and -1 day samples) and the red ellipse represents the samples after FMT ( 3 days, 7 days, 2 weeks, 4 weeks, 8 weeks and 6 months). No separation between the samples before and after FMT was observed in any of the groups.

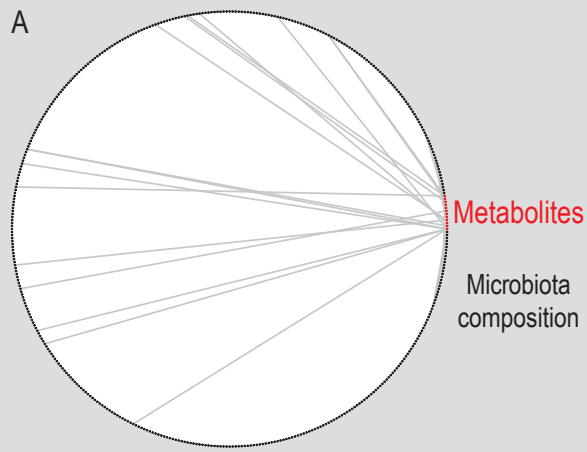

Allogenic FMT

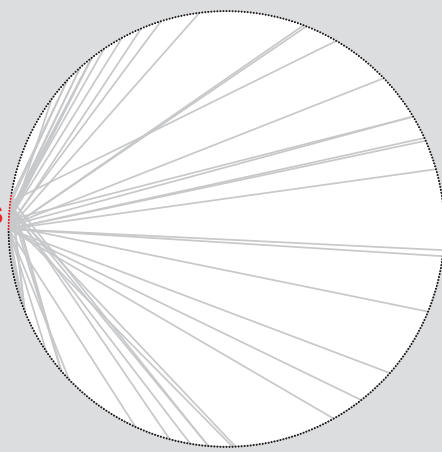

Autologous FMT

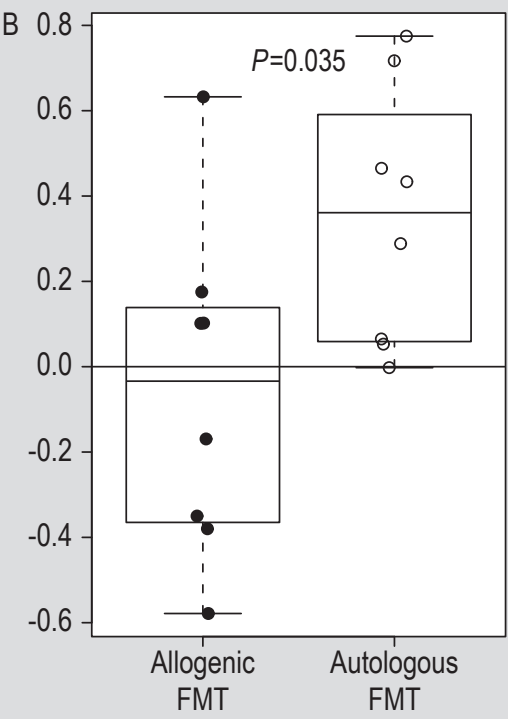

Figure 5. Correlation between metabolites and microbiota composition data. (A) Visualisation of partial correlations between 12 faecal metabolites and the microbiota composition on species level in the allogenic as well as the autologous faecal microbiota transfer (FMT) group. The average of all time points is taken for all individuals in the corresponding groups. The names of the metabolites and the microbial species that correlate can be found in Supplementary Table S2. (B) Canonical correlation analysis performed on the microbial composition data and the measured metabolites. Both analyses suggest that the interactions between microbiota and metabolites were disrupted after allogenic FMT.

allogenic FMT. Similar results were obtained when the measured metabolites were corrected for faecal dry weight (Supplementary Figure S6), and also if different relevance cut-offs for partial correlations were chosen before drawing an interaction line in these plots (data not shown). 


\section{Sparse canonical correlation analysis}

As a multivariate approach to assess correlations between microbiota and specific metabolic profiles, we employed a sparse version of Canonical Correlation Analysis (sCCA), allowing us to deal with a large number of variables compared to samples in our datasets. sCCA analyses were conducted for each subject, in order to relate development of microbiota to changes in metabolite profiles during FMT treatments. Figure 5B shows the multivariate correlation values for each individual in the allogenic and autologous group, which were on group level significantly different from each other $(P=0.035)$. The correlation values in the allogenic FMT group were distributed around zero whereas the values from the autologous FMT group were mostly larger than zero. These results show that the correlations found after allogenic FMT were less homogenous in direction than the correlations found after autologous FMT, indicating again that the interaction between microbiota and metabolites were disrupted after allogenic FMT. The faecal metabolites contributing most to these correlations that were shared by all subjects in the allogenic FMT group included acetic acid and butyric acid. The microbiota contributing the most to these correlations included Clostridium bolteae, Hespellia porcina, Faecalibacterium prausnitzii and Gemmiger formicillis. In the autologous group, acetic acid correlated with Clostridium bolteae and Hespellia porcina. Excluding the six-month samples showed similar results, however no statistically significant difference was reached (Supplementary Figure S7A). The sCCA for dry weight corrected faecal metabolites did not show any differences between allogenic and autologous FMT (Supplementary Figure S7B).

\section{Functional prediction using next generation sequencing data}

Microbiota composition can be used to predict the microbiota's functional potential, based on annotations of reference genomic sequences. We made use of this possibility to produce functional microbiota profiles for all faecal samples from allogenic and autologous FMT treated subjects in order to investigate whether the newly introduced microbiota took over a similar functional capacity as of the original microbiota (using PICRUSt). Figure 6A and B show sPLS analyses results for the functional prediction data. The plots show that the baseline samples separate more clearly from the samples after allogenic FMT compared to autologous FMT. This is similar to the results for the microbiota composition (Figure 1), although to a somewhat lesser extent, which indicates that additionally to the microbiota composition the functional capacity also changes after allogenic FMT. Finally, in order to study the relation between the predicted pathways involved in saccharolytic and proteolytic processes and their metabolites, these pathways and their respective metabolites were correlated (Supplementary Figure S8 and S9). The correlation patterns seem to differ between the allogenic and the autologous group but the correlations are weak and not statistically significant.

\section{Gene expression of metabolite transporters}

A reason for unaltered levels of faecal metabolites in parallel to an altered functional capacity of the microbiota could be an increased uptake of the produced faecal metabolites. FMT could potentially affect the uptake of these metabolites which is often substance-induced. Therefore, the gene expression of several transporters in colonic biopsies before and after FMT was measured with qPCR. While the relative expression of the butyrate and some other transporters was higher after the allogenic than the autologous FMT, no significant differences were observed (Supplementary Figure S10).

\section{Discussion}

FMT is the introduction of a new gut microbiota with the aim to restore a disturbed microbiota. This is the first study, to the best of our knowledge, that provides insight into the interaction between microbial composition and faecal metabolites after the introduction of a foreign microbiota. Especially allogenic FMT (from donor stool) resulted in an altered gut microbiota composition of the recipients even after six months, which is in line with our previously published results where both the faecal and the mucosal gut microbiota, analysed by HITChip, were altered two and eight weeks after FMT (Holster et al., 2019b). However, the measured faecal bacterial metabolites in our study did not seem to be affected by neither allogenic nor autologous FMT. Interestingly though, our data indicate that the correlations between the microbiota and its metabolites appeared to be disrupted after allogenic FMT.

Partial correlations between faecal microbiota and its metabolites showed less correlations in the allogenic FMT group than in the autologous FMT group, which could indicate that the number of functional interactions between microbiota and metabolites is reduced after introducing a new microbiota. Assessing these correlations in a multivariate approach showed that the observed canonical correlations in the allogenic FMT group were less homogeneous in direction than observed in the autologous FMT. In addition to investigating the microbial composition, the 16S rRNA gene based NGS data was used to predict the functional capability of the microbes in the faecal samples. The supervised multivariate analysis showed that the functional capacity of the microbiota after allogenic FMT differed from baseline, similarly to the microbial composition which was altered after allogenic FMT, although to a lesser extent. This could suggest that the change of the microbial ecosystem induced by FMT also 
A

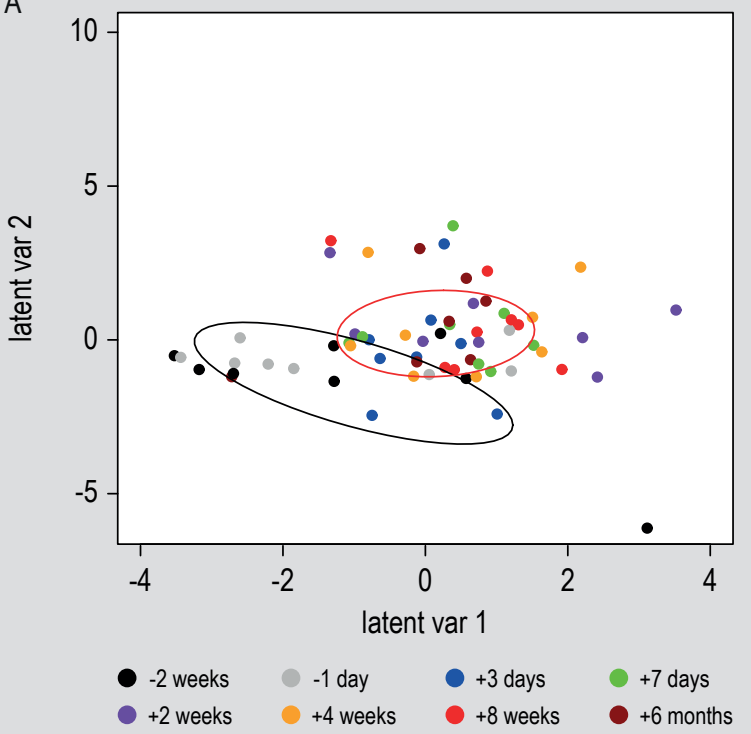

B

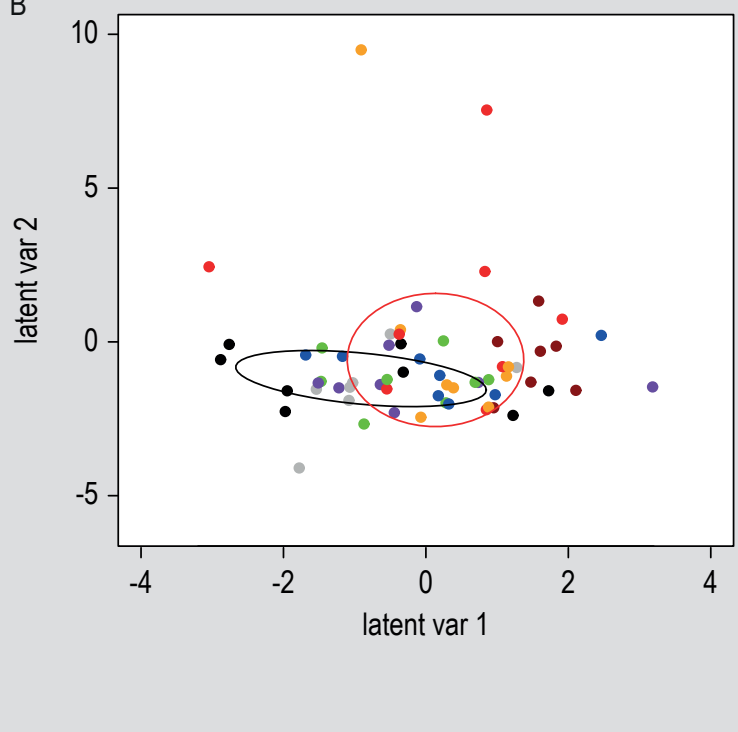

Figure 6. Sparse partial least squares score plots of the functional microbial prediction analysis within the allogenic (A) and autologous group (B) at different time points before and after faecal microbiota transfer (FMT). The first and second latent variable are shown on the $x$ - and $y$-axis. Different colours indicate different time points. Ellipses represent location and dimensions of mean values and covariance structure of the respective part of the data. The black ellipse represents the samples before FMT ( -2 weeks and -1 day samples) and the red ellipse represents the samples after FMT ( 3 days, 7 days, 2 weeks, 4 weeks, 8 weeks and 6 months). The ellipses show that the predicted functional capacity of the faecal microbiota samples were separated from the baseline samples after allogenic FMT, which was not the case after autologous FMT.

changed its overall metabolic capacity but had less impact on the restricted metabolites which were measured in the faecal samples of this study. An additional explanation could be that changes in other microorganisms such as viruses, eukaryotes and fungi had an effect on the metabolic capacity. Furthermore, the microbiota analysis was restricted to $16 \mathrm{~S}$ rRNA-targeted sequencing. Whole genome sequencing could have provided a wider insight into the metabolic capacity of the microbiome.

Regularised methods of data analysis were implemented to assess both univariate and multivariate correlations between microbiota compositional profiles and specific metabolite profiles in the faecal samples during FMT treatment. This was a necessary choice to enable computation of partial correlations as well as canonical correlations, both of which otherwise could not be computed due to our data structure with an excess of variables compared to samples (and time points). Moreover, partial correlations are to be preferred over classical Pearson or Spearman correlations in this case, in order to avoid indirect correlations as far as possible. Partial correlations are frequently used for reverse engineering purposes, to develop hypotheses related to causal interactions (Werhli et al., 2006). Regarding canonical correlation analysis, we looked for specific combinations of microbiota compositional profiles co-varying with combinations of the specific metabolite profiles in faecal samples after FMT. Such analyses allow to investigate more complex interactions between microbiota and metabolite profiles, which would be missed if assessing correlations on single-pairwise levels only.

In our previous study on the clinical effect of FMT in IBS patients, we showed that allogenic FMT did not have a significantly greater effect on symptoms than autologous FMT, although IBS symptoms improved compared to baseline only in the allogenic group (Holster et al., 2019b). However, the mucosal gene expression response was clearly different after allogenic compared to autologous FMT, suggesting an altered microbe-host interaction (Holster et al., 2019a). Bacterial metabolites are an important part of the microbe-host interaction. We hypothesised that changes in bacterial metabolites could have caused the large differences in mucosal response. This would possibly indicate a new mode of action as well as a novel possible therapeutic target. Contrary to our hypothesis, allogenic FMT did not result in an altered metabolite profile in our study.

While faecal SCFA seem to be altered in IBS (Sun et al., 2019), studies investigating other metabolites in IBS are scarce. A study by Shankar et al. (2015) showed that children with IBS-D had a different faecal metabolite profile compared to healthy controls. Additionally, strong associations between microbes and metabolites were found in the healthy children, whereas in the IBS-D group only 
weak associations were detected (Shankar et al., 2015). It seems as if FMT, at least in our study, is not able to restore the weak associations between microbes and metabolites as found in the study by Shankar et al. (2015). However, FMT did result in altered correlations between microbiota composition and faecal metabolites, indicating a change in microbe-metabolite interactions.

A recent study investigated the metabolite profiles post-FMT in IBS patients and concluded that SCFAs normalised after allogenic FMT, however the study did not include a control group and therefore their results cannot be compared to autologous FMT or natural changes occurring over time in IBS patients (Mazzawi et al., 2019). Additionally, in CDI increased levels of the SCFAs butyrate, propionate and acetate were found in six patients after treatment with FMT (Seekatz et al., 2018). Untargeted metabolomics in four paediatric UC patients showed that several metabolites were altered after FMT and correlated with clinical outcomes, and that the patients' metabolomic profile shifted towards their respective donors (Nusbaum et al., 2018). A larger study, which investigated the outcomes of FMT in adult UC patients, suggested that specific metabolomic functional pathways of the microbiota were associated with achieved remission after FMT (Paramsothy et al., 2019).

There are several possible reasons why no changes in metabolites after introducing a foreign microbiota could be observed in our study. First of all, measuring the metabolites in faecal samples does not necessarily reflect the production of bacterial metabolites. Metabolites are taken up by the host, converted into other metabolites or excreted from the host. Although the gene expression of several host mucosal transporters in our study was not affected by FMT, it is still unclear if changes in measured metabolites reflect changes in bacterial production of these metabolites or the ability versus inability of the host to absorb and utilise these metabolites. Therefore, other measures, such as assessing the metabolome in urine and plasma, could give additional insights into the dynamics of the metabolome produced by the gut microbiota.

Another limitation of this study was that only a selective number of metabolites was measured and changes in other metabolites could have been missed. In addition, the autologous FMT group (receiving its own faecal material back) was regarded as a control group, while our earlier publications showed that also after autologous FMT microbial changes were observed and host gene sets were differently expressed. These changes were possibly due to the bowel cleansing before the FMT and/or the handling of the stool in preparation for the FMT. This could have also affected the outcomes measured in this study. In addition, both FMT groups had a limited number of patients not allowing for subgroup analyses. Furthermore, although we asked the participants to maintain their dietary habits while participating in the study and Food Frequency Questionnaire (FFQ) data did not show substantial changes during the study, already small changes in diet could have affected the participants' microbial composition and measured metabolites. However, we expect the effect of FMT to be larger than the effect of possible minor changes in food intake. Finally, although FMT is generally considered a safe treatment with a low incidence of serious adverse effects and the donors used in this were extensively tested, the risk that multi-drug resistant organisms and, possibly, other diseases are transferred seem to be present (DeFilipp et al., 2019). In addition, the long-term risks are not yet fully investigated.

We used the tool PICRUSt for the prediction of functional profiles based on $16 \mathrm{~S}$ rRNA gene date which can provide predicted functional profiles based on pathway or protein level. The small sample size of our study only allowed for such profiles on pathway level, while a larger samples size would have enabled more accurate profiles based on protein level. In addition, PICRUSt is one of several choices of methods for predicting functional profiles, and the use of alternative pipelines would very likely have resulted in differences in data analysis results. Given the limited sample size of our study population and the known large variation in microbiota composition between different individuals, we could not deliver detailed results on any specific predicted functional profile, but instead constrained our conclusions to overall measures of correlation patterns, such as total numbers of observed partial correlations, or intensities of observed canonical correlations, between measured faecal metabolites and microbiota composition or predicted functional profiles. In addition, at least in our study, it seems as if using prediction of metabolic function based on microbiota composition analysis does not seem to be a good surrogate marker for the faecal metabolites that were measured.

In conclusion, we could show that FMT resulted in disturbed interactions between the microbiota and its metabolites in IBS. Future studies need to elucidate how this affects treatment outcome in FMT therapy, especially with regards to personalised medicine.

\section{Supplementary material}

Supplementary material can be found online at https://doi. org/10.3920/BM2020.0010.

Table S1. The quantification ions in the GC-MS method.

Table S2. Partial correlations between metabolites and microbiota composition data in the allogenic FMT and autologous FMT group. 
Figure S1. Top 10 most abundant genera in faecal samples of IBS patients included in this study.

Figure S2. sPLS figures of faecal microbial composition within the allogenic group and autologous group at different time points before and after FMT without six months sample.

Figure S3. Absolute data of the twelve metabolites measured in the faecal material before and after allogenic or autologous FMT in IBS patients.

Figure S4. Baseline-corrected data of the twelve metabolites measured in the faecal material before and after allogenic or autologous FMT in IBS patients.

Figure S5. sPLS figures of measured metabolites normalised to dry weight within the allogenic group and autologous group at different time points before and after FMT Ellipses represent location and dimensions of mean values and covariance structure of the respective part of the data.

Figure S6. Correlation between metabolites corrected for faecal dry weight and microbiota composition data.

Figure S7. Canonical correlation analysis performed on the microbial composition data and the measured metabolites and the microbial composition data and metabolites corrected for dry weight without the six-month sample.

Figure S8. Heatmap of correlations between the predicted pathways involved in saccharolytic processes and their metabolites in the allogenic group and autologous group.

Figure S9. Heatmap of correlations between the predicted pathways involved in proteolytic processes and their metabolites in the allogenic group and autologous group.

Figure S10. Relative expression of several transporters in biopsies of IBS patients after allogenic or autologous FMT, 2 and 8 weeks after FMT compared to baseline expression.

\section{Acknowledgements}

The authors would like to thank Rebecca Wall and Michiel Diender for their help with the RNA isolation and qPCR. We are grateful to Tinja Kanerva and Evgenia Dikareva for supporting the NGS pipeline. Part of this work was funded by a grant of the Knowledge Foundation to the Nutrition-Gut-Brain Interactions Research Centre, as well as a research fellowship awarded to Julia König by the European Society of Clinical Nutrition and Metabolism (ESPEN). This research was also partly supported by the Netherlands Organization for Scientific Research (Spinoza Award to WMdV).

\section{Conflict of interest}

The authors declare no conflict of interest.

\section{References}

Aitchison, J. and Greenacre, M., 2002. Biplots of compositional data. Journal of the Royal Statistical Society Series C-Applied Statistics 51: 375-392. https://doi.org/10.1111/1467-9876.00275

Cao, K.A.L., Rohart, F., Gonzalez, I., Dejean, S., Gautier, B., Bartolo, F., Monget, P., Coquery, J., Yao, F. and Liquet, B., 2017. mixOmics: omics data integration project. Available at: https://CRAN.R-project. org $/$ package $=$ mixOmics

Comas-Cufi, M., 2019. coda.base: a basic set of functions for compositional data analysis. Available at: https://CRAN.R-project. org $/$ package $=$ coda.base

Correa-Oliveira, R., Fachi, J.L., Vieira, A., Sato, F.T. and Vinolo, M.A., 2016. Regulation of immune cell function by short-chain fatty acids. Clinical and Translational Immunology 5: e73. https://doi. org/10.1038/cti.2016.17

Costello, S.P., Hughes, P.A., Waters, O., Bryant, R.V., Vincent, A.D., Blatchford, P., Katsikeros, R., Makanyanga, J., Campaniello, M.A., Mavrangelos, C., Rosewarne, C.P., Bickley, C., Peters, C., Schoeman, M.N., Conlon, M.A., Roberts-Thomson, I.C. and Andrews, J.M., 2019. Effect of fecal microbiota transplantation on 8-week remission in patients with ulcerative colitis: a randomized clinical trial. JAMA 321: 156-164. https://doi.org/10.1001/jama.2018.20046

Dai, Z.L., Wu, G. and Zhu, W.Y., 2011. Amino acid metabolism in intestinal bacteria: links between gut ecology and host health. Frontiers in Bioscience 16: 1768-1786. https://doi.org/10.2741/3820

De Vos, W.M., 2013. Fame and future of faecal transplantations developing next-generation therapies with synthetic microbiomes. Microbial Biotechnology 6: 316-325. https://doi.org/10.1111/17517915.12047

DeFilipp, Z., Bloom, P.P., Torres Soto, M., Mansour, M.K., Sater, M.R.A., Huntley, M.H., Turbett, S., Chung, R.T., Chen, Y.B. and Hohmann, E.L., 2019. Drug-resistant E. coli bacteremia transmitted by fecal microbiota transplant. New England Journal of Medicine 381: 2043-2050. https://doi.org/10.1056/NEJMoa1910437

Durban, A., Abellan, J.J., Jimenez-Hernandez, N., Artacho, A., Garrigues, V., Ortiz, V., Ponce, J., Latorre, A. and Moya, A., 2013. Instability of the faecal microbiota in diarrhoea-predominant irritable bowel syndrome. FEMS Microbiology Ecology 86: 581589. https://doi.org/10.1111/1574-6941.12184

Halkjaer, S.I., Christensen, A.H., Lo, B.Z.S., Browne, P.D., Gunther, S., Hansen, L.H. and Petersen, A.M., 2018. Faecal microbiota transplantation alters gut microbiota in patients with irritable bowel syndrome: results from a randomised, double-blind placebocontrolled study. Gut 67: 2107-2115. https://doi.org/10.1136/ gutjnl-2018-316434

Holster, S., Hooiveld, G.J., Repsilber, D., Vos, W.M., Brummer, R.J. and König, J., 2019a. Allogenic faecal microbiota transfer induces immune-related gene sets in the colon mucosa of patients with irritable bowel syndrome. Biomolecules 9: 586. https://doi. org/10.3390/biom 9100586 
Holster, S., Lindqvist, C.M., Repsilber, D., Salonen, A., De Vos, W.M., König, J. and Brummer, R.J., 2019b. The effect of allogenic versus autologous fecal microbiota transfer on symptoms, visceral perception and fecal and mucosal microbiota in irritable bowel syndrome: a randomized controlled study. Clinical and Translational Gastroenterology 10: e00034. https://doi.org/10.14309/ ctg.0000000000000034

Hout, M.C., Papesh, M.H. and Goldinger, S.D., 2013. Multidimensional scaling. Wiley Interdisciplinary Reviews: Cognitive Science 4: 93103. https://doi.org/10.1002/wcs.1203

Johnsen, P.H., Hilpusch, F., Cavanagh, J.P., Leikanger, I.S., Kolstad, C., Valle, P.C. and Goll, R., 2018. Faecal microbiota transplantation versus placebo for moderate-to-severe irritable bowel syndrome: a double-blind, randomised, placebo-controlled, parallel-group, single-centre trial. The Lancet Gastroenterology and Hepatology 3: 17-24. https://doi.org/10.1016/S2468-1253(17)30338-2

Kanehisa, M. and Goto, S., 2000. KEGG: kyoto encyclopedia of genes and genomes. Nucleic Acids Research 28: 27-30. https:// doi.org/10.1093/nar/28.1.27

Kenkel, N.C. and Orloci, L., 1986. Applying metric and nonmetric multidimensional-scaling to ecological-studies - some new results. Ecology 67: 919-928. https://doi.org/10.2307/1939814

Kootte, R.S., Levin, E., Salojarvi, J., Smits, L.P., Hartstra, A.V., Udayappan, S.D., Hermes, G., Bouter, K.E., Koopen, A.M., Holst, J.J., Knop, F.K., Blaak, E.E., Zhao, J., Smidt, H., Harms, A.C., Hankemeijer, T., Bergman, J., Romijn, H.A., Schaap, F.G., Olde Damink, S.W.M., Ackermans, M.T., Dallinga-Thie, G.M., Zoetendal, E., De Vos, W.M., Serlie, M.J., Stroes, E.S.G., Groen, A.K. and Nieuwdorp, M., 2017. Improvement of insulin sensitivity after lean donor feces in metabolic syndrome is driven by baseline intestinal microbiota composition. Cell Metabolism 26: 611-619. https://doi. org/10.1016/j.cmet.2017.09.008

Lahtinen, P., Jalanka, J., Hartikainen, A., Mattila, E., Hillila, M., Punkkinen, J., Koskenpato, J., Anttila, V.J., Tillonen, J., Satokari, R. and Arkkila, P., 2020. Randomised clinical trial: faecal microbiota transplantation versus autologous placebo administered via colonoscopy in irritable bowel syndrome. Alimentary Pharmacology and Therapeutics 51: 1321-1331. https://doi.org/10.1111/apt.15740

Langille, M.G., Zaneveld, J., Caporaso, J.G., McDonald, D., Knights, D., Reyes, J.A., Clemente, J.C., Burkepile, D.E., Vega Thurber, R.L., Knight, R., Beiko, R.G. and Huttenhower, C., 2013. Predictive functional profiling of microbial communities using $16 \mathrm{~S}$ rRNA marker gene sequences. Nature Biotechnology 31: 814-821. https:// doi.org/10.1038/nbt.2676

Levy, M., Thaiss, C.A. and Elinav, E., 2016. Metabolites: messengers between the microbiota and the immune system. Genes and Development 30: 1589-1597. https://doi.org/10.1101/gad.284091.116

Li, S.S., Zhu, A., Benes, V., Costea, P.I., Hercog, R., Hildebrand, F., Huerta-Cepas, J., Nieuwdorp, M., Salojarvi, J., Voigt, A.Y., Zeller, G., Sunagawa, S., De Vos, W.M. and Bork, P., 2016. Durable coexistence of donor and recipient strains after fecal microbiota transplantation. Science 352: 586-589. https://doi.org/10.1126/science.aad8852

Lin, R., Liu, W., Piao, M. and Zhu, H., 2017. A review of the relationship between the gut microbiota and amino acid metabolism. Amino Acids 49: 2083-2090. https://doi.org/10.1007/s00726-017-2493-3
Livak, K.J. and Schmittgen, T.D., 2001. Analysis of relative gene expression data using real-time quantitative PCR and the 2(-Delta Delta C(T)) method. Methods 25: 402-408. https://doi.org/10.1006/ meth.2001.1262

Matto, J., Maunuksela, L., Kajander, K., Palva, A., Korpela, R., Kassinen, A. and Saarela, M., 2005. Composition and temporal stability of gastrointestinal microbiota in irritable bowel syndrome - a longitudinal study in IBS and control subjects. FEMS Immunology and Medical Microbiology 43: 213-222. https://doi.org/10.1016/j. femsim.2004.08.009

Mazzawi, T., Hausken, T., Hov, J.R., Valeur, J., Sangnes, D.A., ElSalhy, M., Gilja, O.H., Hatlebakk, J.G. and Lied, G.A., 2019. Clinical response to fecal microbiota transplantation in patients with diarrhea-predominant irritable bowel syndrome is associated with normalization of fecal microbiota composition and short-chain fatty acid levels. Scandinavian Journal of Gastroenterology 54: 690-699. https://doi.org/10.1080/00365521.2019.1624815

Moayyedi, P., Surette, M.G., Kim, P.T., Libertucci, J., Wolfe, M., Onischi, C., Armstrong, D., Marshall, J.K., Kassam, Z., Reinisch, W. and Lee, C.H., 2015. Fecal microbiota transplantation induces remission in patients with active ulcerative colitis in a randomized controlled trial. Gastroenterology 149: 102-109. https://doi.org/10.1053/j. gastro.2015.04.001

Navas-Molina, J.A., Peralta-Sanchez, J.M., Gonzalez, A., McMurdie, P.J., Vazquez-Baeza, Y., Xu, Z.J., Ursell, L.K., Lauber, C., Zhou, H.W., Song, S.J., Huntley, J., Ackermann, G.L., Berg-Lyons, D., Holmes, S., Caporaso, J.G. and Knight, R., 2013. Advancing our understanding of the human microbiome using QIIME. Microbial Metagenomics, Metatranscriptomics, and Metaproteomics 531: 371-444. https:// doi.org/10.1016/B978-0-12-407863-5.00019-8

Neis, E.P., Dejong, C.H. and Rensen, S.S., 2015. The role of microbial amino acid metabolism in host metabolism. Nutrients 7: 2930-2946. https://doi.org/10.3390/nu7042930

Nusbaum, D.J., Sun, F., Ren, J., Zhu, Z., Ramsy, N., Pervolarakis, N., Kunde, S., England, W., Gao, B., Fiehn, O., Michail, S. and Whiteson, K., 2018. Gut microbial and metabolomic profiles after fecal microbiota transplantation in pediatric ulcerative colitis patients. FEMS Microbiology Ecology 94: fiy133. https://doi.org/10.1093/ femsec/fiy133

Paramsothy, S., Kamm, M.A., Kaakoush, N.O., Walsh, A.J., Van den Bogaerde, J., Samuel, D., Leong, R.W.L., Connor, S., Ng, W., Paramsothy, R., Xuan, W., Lin, E., Mitchell, H.M. and Borody, T.J., 2017. Multidonor intensive faecal microbiota transplantation for active ulcerative colitis: a randomised placebo-controlled trial. The Lancet 389: 1218-1228. https://doi.org/10.1016/S01406736(17)30182-4

Paramsothy, S., Nielsen, S., Kamm, M.A., Deshpande, N.P., Faith, J.J., Clemente, J.C., Paramsothy, R., Walsh, A.J., Van den Bogaerde, J., Samuel, D., Leong, R.W.L., Connor, S., Ng, W., Lin, E., Borody, T.J., Wilkins, M.R., Colombel, J.F., Mitchell, H.M. and Kaakoush, N.O., 2019. Specific bacteria and metabolites associated with response to fecal microbiota transplantation in patients with ulcerative colitis. Gastroenterology 156: 1440-1454 e1442. https://doi.org/10.1053/j. gastro.2018.12.001 
Pedersen, H.K., Gudmundsdottir, V., Nielsen, H.B., Hyotylainen, T., Nielsen, T., Jensen, B.A., Forslund, K., Hildebrand, F., Prifti, E., Falony, G., Le Chatelier, E., Levenez, F., Dore, J., Mattila, I., Plichta, D.R., Poho, P., Hellgren, L.I., Arumugam, M., Sunagawa, S., Vieira-Silva, S., Jorgensen, T., Holm, J.B., Trost, K., Meta, H.I.T.C., Kristiansen, K., Brix, S., Raes, J., Wang, J., Hansen, T., Bork, P., Brunak, S., Oresic, M., Ehrlich, S.D. and Pedersen, O., 2016. Human gut microbes impact host serum metabolome and insulin sensitivity. Nature 535: 376-381. https://doi.org/10.1038/nature18646

Pittayanon, R., Lau, J.T., Yuan, Y., Leontiadis, G.I., Tse, F., Surette, M. and Moayyedi, P., 2019. Gut microbiota in patients with irritable bowel syndrome-a systematic review. Gastroenterology 157: 97-108. https://doi.org/10.1053/j.gastro.2019.03.049

Quraishi, M.N., Widlak, M., Bhala, N., Moore, D., Price, M., Sharma, N. and Iqbal, T.H., 2017. Systematic review with meta-analysis: the efficacy of faecal microbiota transplantation for the treatment of recurrent and refractory Clostridium difficile infection. Alimentary Pharmacology and Therapeutics 46: 479-493. https:// doi.org/10.1111/apt.14201

Rohart, F., Gautier, B., Singh, A. and Le Cao, K.A., 2017. mixOmics: an $\mathrm{R}$ package for 'omics feature selection and multiple data integration. PLoS Computational Biology 13: e1005752. https://doi.org/10.1371/ journal.pcbi.1005752

Rossen, N.G., Fuentes, S., Van der Spek, M.J., Tijssen, J.G., Hartman, J.H., Duflou, A., Lowenberg, M., Van den Brink, G.R., MathusVliegen, E.M., De Vos, W.M., Zoetendal, E.G. D’Haens, G.R. and Ponsioen, C.Y., 2015. Findings from a randomized controlled trial of fecal transplantation for patients with ulcerative colitis. Gastroenterology 149: 110-118. https://doi.org/10.1053/j. gastro.2015.03.045

Salonen, A., Nikkila, J., Jalanka-Tuovinen, J., Immonen, O., RajilicStojanovic, M., Kekkonen, R.A., Palva, A. and De Vos, W.M., 2010. Comparative analysis of fecal DNA extraction methods with phylogenetic microarray: effective recovery of bacterial and archaeal DNA using mechanical cell lysis. Journal of Microbiological Methods 81: 127-134. https://doi.org/10.1016/j.mimet.2010.02.007

Schafer, J., Opgen-Rhein, R., Zuber, V., Ahdesmaki, M., Silva, A.P.D. and Strimmer, K., 2017. corpcor: efficient estimation of covariance and (partial) correlation. Available at: https://CRAN.R-project.org/ package $=$ corpcor

Schafer, J. and Strimmer, K., 2005. A shrinkage approach to largescale covariance matrix estimation and implications for functional genomics. Statistical Applications in Genetics and Molecular Biology 4: 32. https://doi.org/10.2202/1544-6115.1175

Schicho, R., Shaykhutdinov, R., Ngo, J., Nazyrova, A., Schneider, C., Panaccione, R., Kaplan, G.G., Vogel, H.J. and Storr, M., 2012. Quantitative metabolomic profiling of serum, plasma, and urine by (1)H NMR spectroscopy discriminates between patients with inflammatory bowel disease and healthy individuals. Journal of Proteome Research 11: 3344-3357. https://doi.org/10.1021/ pr300139q
Schloss, P.D., Westcott, S.L., Ryabin, T., Hall, J.R., Hartmann, M., Hollister, E.B., Lesniewski, R.A., Oakley, B.B., Parks, D.H., Robinson, C.J., Sahl, J.W., Stres, B., Thallinger, G.G., Van Horn, D.J. and Weber, C.F., 2009. Introducing mothur: open-source, platform-independent, community-supported software for describing and comparing microbial communities. Applied and Environmental Microbiology 75: 7537-7541. https://doi.org/10.1128/AEM.01541-09

Scott, K.P., Gratz, S.W., Sheridan, P.O., Flint, H.J. and Duncan, S.H., 2013. The influence of diet on the gut microbiota. Pharmacological Research 69: 52-60. https://doi.org/10.1016/j.phrs.2012.10.020

Seekatz, A.M., Theriot, C.M., Rao, K., Chang, Y.M., Freeman, A.E., Kao, J.Y. and Young, V.B., 2018. Restoration of short chain fatty acid and bile acid metabolism following fecal microbiota transplantation in patients with recurrent Clostridium difficile infection. Anaerobe 53: 64-73. https://doi.org/10.1016/j.anaerobe.2018.04.001

Shankar, V., Homer, D., Rigsbee, L., Khamis, H.J., Michail, S., Raymer, M., Reo, N.V. and Paliy, O., 2015. The networks of human gut microbe-metabolite associations are different between health and irritable bowel syndrome. ISME Journal 9: 1899-1903. https://doi. org/10.1038/ismej.2014.258

Sun, Q., Jia, Q., Song, L. and Duan, L., 2019. Alterations in fecal short-chain fatty acids in patients with irritable bowel syndrome: a systematic review and meta-analysis. Medicine 98: e14513. https:// doi.org/10.1097/MD.0000000000014513

Van der Beek, C.M., Dejong, C.H.C., Troost, F.J., Masclee, A.A.M. and Lenaerts, K., 2017. Role of short-chain fatty acids in colonic inflammation, carcinogenesis, and mucosal protection and healing. Nutrition Reviews 75: 286-305. https://doi.org/10.1093/nutrit/ nuw067

Vanhoutvin, S.A., Troost, F.J., Kilkens, T.O., Lindsey, P.J., Hamer, H.M., Jonkers, D.M., Venema, K. and Brummer, R.J., 2009. The effects of butyrate enemas on visceral perception in healthy volunteers. Neurogastroenterology and Motility 21: 952-e976. https://doi. org/10.1111/j.1365-2982.2009.01324.x

Virtanen, S., Rantsi, T., Virtanen, A., Kervinen, K., Nieminen, P., Kalliala, I. and Salonen, A., 2019. Vaginal microbiota composition correlates between pap smear microscopy and next generation sequencing and associates to socioeconomic status. Scientific Reports 9: 7750. https://doi.org/10.1038/s41598-019-44157-8

Vrieze, A., Van Nood, E., Holleman, F., Salojarvi, J., Kootte, R.S., Bartelsman, J.F., Dallinga-Thie, G.M., Ackermans, M.T., Serlie, M.J., Oozeer, R., Derrien, M., Druesne, A., Van Hylckama Vlieg, J.E., Bloks, V.W., Groen, A.K., Heilig, H.G., Zoetendal, E.G. Stroes, E.S., De Vos, W.M., Hoekstra, J.B. and Nieuwdorp, M., 2012. Transfer of intestinal microbiota from lean donors increases insulin sensitivity in individuals with metabolic syndrome. Gastroenterology 143: 913-916. https://doi.org/10.1053/j.gastro.2012.06.031

Werhli, A.V., Grzegorczyk, M. and Husmeier, D., 2006. Comparative evaluation of reverse engineering gene regulatory networks with relevance networks, graphical gaussian models and bayesian networks. Bioinformatics 22: 2523-2531. https://doi.org/10.1093/ bioinformatics/btl391

Witten, D.M., Tibshirani, R. and Hastie, T., 2009. A penalized matrix decomposition, with applications to sparse principal components and canonical correlation analysis. Biostatistics 10: 515-534. https:// doi.org/10.1093/biostatistics/kxp008 
Wu, X., Wu, Y., He, L., Wu, L., Wang, X. and Liu, Z., 2018. Effects of the intestinal microbial metabolite butyrate on the development of colorectal cancer. Journal of Cancer 9: 2510-2517. https://doi. org/10.7150/jca.25324
Yang, Y., Misra, B.B., Liang, L., Bi, D., Weng, W., Wu, W., Cai, S., Qin, H., Goel, A., Li, X. and Ma, Y., 2019. Integrated microbiome and metabolome analysis reveals a novel interplay between commensal bacteria and metabolites in colorectal cancer. Theranostics 9: 41014114. https://doi.org/10.7150/thno.35186 